\title{
ON INTERIOR LAYERS FOR INTEGRAL EQUATIONS
}

\author{
Ren-ji Sun
}

\begin{abstract}
A class of Fredholm integral equations is considered and the boundary layer correction method is developed with an interior layer. An analysis of the residual and some examples are presented.
\end{abstract}

\section{Introduction}

Boundary layer methods are well-known and are used to analyze the singular perturbation problems of differential equations [1], [2]. However, solutions to integral equations with boundary layer behavior are more difficult since the "outer" and "inner" contributions directly influence each other; they do not separate as easily as they do for differential equations. Naive analyses introduce divergent integrals, which cause trouble. Recently, Lange and Smith [3] and Olmstead and Angell [4] applied the boundary layer correction method to analyze the boundary layer problems for integral equations. The boundary layers they analyze are located at the end points of the integral. In the present paper, we consider integral equations with an interior layer and establish a solution method. An analysis of the residual is presented. In the end some examples are given. The composite expansion will be the same as that obtained by using the method of steepest descent.

\section{The perturbation method}

In this paper we consider the following scalar Fredholm integral equation

$$
\varepsilon u(x)+h(x, \varepsilon)=\int_{0}^{1} K(x, s) u(s) d s,
$$

where $\varepsilon$ is a small positive parameter $\left(\varepsilon \rightarrow 0^{+}\right)$. The kernel is assumed discontinuous at $x=s$, that is

$$
K(x, s)= \begin{cases}L(x, s), & s<x \\ R(x, s), & s>x\end{cases}
$$

where $L(x, s)$ and $R(x, s)$ are sufficiently smooth functions on $[0,1] \times[0,1]$. The "jump" in the kernel

$$
J(x)=L(x, x)-R(x, x)
$$

Received April 5, 1993, revised February 23, 1994.

1991 Mathematics Subject Classification: 45L05, 45B05.

Key words and phrases: singular perturbation, integral equations. 
is zero only at $x=x_{0}$, an internal point which will be the location of the interior layer. The forcing function $h(x, \varepsilon)$ is smooth and has an asymptotic expansion

$$
h(x, \varepsilon) \sim \sum_{j=0}^{\infty} h_{j}(x) \varepsilon^{j / 2},
$$

where $h_{j}(x)$ are sufficiently smooth functions.

We assume that (1.1) possesses a unique solution which has the following composite asymptotic expansion

$$
u(x) \sim w(x)+\alpha(\varepsilon) B(Z) \quad\left(\varepsilon \rightarrow 0^{+}\right),
$$

where the outer solution $w(x)$ is of the order unity. The "boundary layer correction" $B(Z)$ possesses the property that it will decay quickly as $Z \rightarrow \pm \infty$. That is, it will satisfy

$$
B(Z)=o\left(Z^{-k}\right), Z \rightarrow \pm \infty, \quad k=0,1,2, \ldots
$$

$Z$ is the inner variable of the interior layer:

$$
Z=\left(x-x_{0}\right) / \rho(\varepsilon)
$$

where $x_{0}$ is the position where the interior layer is located. Here $\rho(\varepsilon)$ is the width of the interior layer and $\alpha(\varepsilon)$ is the scale of the "boundary layer correction". By substituting (1.5) into (1.1) we obtain

$$
\begin{aligned}
\varepsilon[w(x)+\alpha(\varepsilon) B(Z)]+h(x, \varepsilon)= & \int_{0}^{x} L(x, s)[w(s)+\alpha(\varepsilon) B(S)] d s \\
& +\int_{x}^{1} R(x, s)[w(s)+\alpha(\varepsilon) B(S)] d s
\end{aligned}
$$

where $S=\left(s-x_{0}\right) / \rho(\varepsilon)$. We assume the "boundary layer" is located in the interior, $x_{0} \in(0,1)$. The method of Lange and Smith [3] for end point boundary layers will be modified somewhat to account for an interior layer.

The outer solution $w(x)$ for $x<x_{0}$ satisfies the following equation

$$
\begin{aligned}
\varepsilon w(x)+h(x, \varepsilon) \sim \int_{0}^{1} K(x, s) w(s) d s & \\
& +\rho(\varepsilon) \alpha(\varepsilon) \int_{-\infty}^{+\infty} R\left(x, x_{0}+\rho(\varepsilon) S\right) B(S) d s
\end{aligned}
$$

since in the outer region,

$$
\begin{gathered}
\varepsilon \alpha(\varepsilon) B(Z) \sim o\left(\varepsilon^{k}\right), \quad k=0,1,2, \ldots \\
\alpha(\varepsilon) \int_{0}^{x} L(x, s) B(S) d s \sim o\left(\varepsilon^{k}\right), \quad k=0,1,2, \ldots, \\
\alpha(\varepsilon) \int_{x}^{1} R(x, s) B(S) d s \sim \rho(\varepsilon) \alpha(\varepsilon) \int_{-\infty}^{+\infty} R\left(x, x_{0}+\rho(\varepsilon) S\right) B(S) d S .
\end{gathered}
$$


A similar equation is valid for $x>x_{0}$ :

$$
\begin{aligned}
\varepsilon w(x)+h(x, \varepsilon) \sim \int_{0}^{1} K(x, s) w(s) d s & \\
& +\rho(\varepsilon) \alpha(\varepsilon) \int_{-\infty}^{+\infty} L\left(x, x_{0}+\rho(\varepsilon) S\right) B(S) d S
\end{aligned}
$$

Consequently, if $R\left(x, x_{0}\right) \neq 0$, we obtain

$$
\alpha(\varepsilon)=[\rho(\varepsilon)]^{-1} .
$$

Taking the limit $x \rightarrow x_{0}$ in (1.8) and (1.9) yields

$$
\int_{-\infty}^{+\infty} R\left(x, x_{0}+\rho(\varepsilon) S\right) B(S) d S=\int_{-\infty}^{+\infty} L\left(x, x_{0}+\rho(\varepsilon) S\right) B(S) d S
$$

Equation (1.11) determines $x_{0}$, where the interior layer is located.

The "boundary layer correction" $B(Z)$ satisfies the asymptotic equation determined by substituting (1.8) and (1.9) into (1.7), using the change of variable $s=x_{0}+\rho(\varepsilon) S$,

$$
\begin{aligned}
\varepsilon B(Z) \sim \rho(\varepsilon) & \int_{-\infty}^{Z}\left[L\left(x_{0}+\rho(\varepsilon) Z, x_{0}+\rho(\varepsilon) S\right)\right. \\
& \left.-R\left(x_{0}+\rho(\varepsilon) Z, x_{0}+\rho(\varepsilon) S\right)\right] B(S) d S
\end{aligned}
$$

for $x<x_{0}$, and

$$
\begin{aligned}
\varepsilon B(Z) \sim \rho(\varepsilon) & \int_{Z}^{+\infty}\left[R\left(x_{0}+\rho(\varepsilon) Z, x_{0}+\rho(\varepsilon) S\right)\right. \\
& \left.-L\left(x_{0}+\rho(\varepsilon) Z, x_{0}+\rho(\varepsilon) S\right)\right] B(S) d S
\end{aligned}
$$

for $x>x_{0}$. By expanding $R$ and $L$ in (1.12) and (1.13) in Taylor series and considering (1.11) we obtain

$$
\begin{aligned}
\varepsilon B(Z) \sim & \int_{-\infty}^{Z} \sum_{n=0}^{\infty}[1 /(n+1) !][\rho(\varepsilon)]^{n+2} \\
& {\left[Z \frac{\partial}{\partial x}+S \frac{\partial}{\partial s}\right]^{n+1}\left[L\left(x_{0}, x_{0}\right)-R\left(x_{0}, x_{0}\right)\right] B(S) d S }
\end{aligned}
$$

for $x<x_{0}$, and

$$
\begin{aligned}
\varepsilon B(Z) \sim & \int_{Z}^{+\infty}[1 /(n+1) !] \sum_{n=0}^{\infty}[\rho(\varepsilon)]^{n+2} \\
& {\left[Z \frac{\partial}{\partial x}+S \frac{\partial}{\partial x}\right]^{n+1}\left[R\left(x_{0}, x_{0}\right)-L\left(x_{0}, x_{0}\right)\right] B(S) d S }
\end{aligned}
$$

for $x>x_{0}$. If

$$
\left(Z \frac{\partial}{\partial x}+S \frac{\partial}{\partial s}\right)\left[L\left(x_{0}, x_{0}\right)-R\left(x_{0}, x_{0}\right)\right] \neq 0
$$

then we have

$$
\rho^{2}(\varepsilon)=\varepsilon \quad \text { or } \quad \rho(\varepsilon)=\varepsilon^{1 / 2}
$$


From (1.10) we have

$$
\alpha(\varepsilon)=\varepsilon^{-1 / 2}
$$

We expand $w(x)$ and $B(Z)$ in asymptotic series of $\varepsilon^{1 / 2}$ :

$$
\begin{aligned}
& w(x, \varepsilon)=u_{0}(x)+\varepsilon^{1 / 2} u_{1}(x)+\cdots, \\
& B(Z)=B_{0}(Z)+\varepsilon^{1 / 2} B_{1}(Z)+\cdots .
\end{aligned}
$$

The equations for $u_{0}$ and $B_{0}$ are obtained by substituting (1.19) and (1.20) in (1.8), (1.9), (1.14) and (1.15). If $x<x_{0}$, then

$$
\begin{gathered}
h_{0}(x)=\int_{0}^{1} K(x, s) u_{0}(s) d s+\int_{-\infty}^{+\infty} R\left(x, x_{0}\right) B_{0}(S) d S \\
B_{0}(Z)=\int_{-\infty}^{Z}\left(Z \frac{\partial}{\partial x}+S \frac{\partial}{\partial s}\right)\left[L\left(x_{0}, x_{0}\right)-R\left(x_{0}, x_{0}\right)\right] B_{0}(S) d S .
\end{gathered}
$$

If $x>x_{0}$, then

$$
\begin{gathered}
h_{0}(x)=\int_{0}^{1} K(x, s) u_{0}(s) d s+\int_{-\infty}^{+\infty} L\left(x, x_{0}\right) B_{0}(S) d S \\
B_{0}(Z)=\int_{Z}^{+\infty}\left(Z \frac{\partial}{\partial x}+S \frac{\partial}{\partial s}\right)\left[R\left(x_{0}, x_{0}\right)-L\left(x_{0}, x_{0}\right)\right] B_{0}(S) d S .
\end{gathered}
$$

We introduce the notation that a prime with subscript $i(i=1,2)$ represents the partial derivative with respect to the $i$-th variable in the functions. If both equations (1.21) and (1.23) have a unique solution and if

$$
J^{\prime}\left(x_{0}\right)=\left[L_{1}^{\prime}\left(x_{0}, x_{0}\right)-R_{1}^{\prime}\left(x_{0}, x_{0}\right)\right]-\left[L_{2}^{\prime}\left(x_{0}, x_{0}\right)-R_{2}^{\prime}\left(x_{0}, x_{0}\right)\right]<0,
$$

then (1.22) or (1.24) possesses the following asymptotic solutions:

$$
\begin{aligned}
& B_{0}(Z) \sim c \exp \left\{J^{\prime}\left(x_{0}\right) Z^{2} / 2\right. \\
& \left.+\ln \left[L_{1}^{\prime}\left(x_{0}, x_{0}\right)-R_{1}^{\prime}\left(x_{0}, x_{0}\right)\right]-\ln \left[J^{\prime}\left(x_{0}\right)\right]\right\}, \quad Z \rightarrow \pm \infty,
\end{aligned}
$$

where $c$ is a constant which can be determined by substituting (1.26) into (1.21) or (1.23).

The derivative of $h_{0}(x)$ is determined from (1.21):

$$
h_{0}^{\prime}(x)=J(x) u_{0}(x)+\int_{0}^{1} K_{1}^{\prime}(x, s) u_{0}(s) d s-\int_{-\infty}^{+\infty} R_{1}^{\prime}\left(x, x_{0}\right) B_{0}(S) d S .
$$

By eliminating $\int_{-\infty}^{+\infty} B_{0}(S) d S$ in (1.21) and (1.27) we obtain

$$
\begin{aligned}
J(x) u_{0}(x)-[ & \left.h_{0}^{\prime}(x)-R_{1}^{\prime}\left(x, x_{0}\right) h_{0}(x) / R\left(x, x_{0}\right)\right] \\
= & \int_{0}^{x}\left[R_{1}^{\prime}\left(x, x_{0}\right) L(x, s) / R\left(x, x_{0}\right)-L_{1}^{\prime}(x, s)\right] u_{0}(s) d s \\
& \quad+\int_{x}^{1}\left[R_{1}^{\prime}\left(x, x_{0}\right) R(x, s) / R\left(x, x_{0}\right)-R_{1}^{\prime}(x, s)\right] u_{0}(s) d s
\end{aligned}
$$


for $x<x_{0}$. Similarly, if we start from (1.22), we obtain

$$
\begin{aligned}
& J(x) u_{0}(x)-\left[h_{0}^{\prime}(x)-L_{1}^{\prime}\left(x, x_{0}\right) h_{0}(x) / L\left(x, x_{0}\right)\right] \\
& =\int_{0}^{x}\left[L_{1}^{\prime}\left(x, x_{0}\right) L(x, s) / L\left(x, x_{0}\right)-L_{1}^{\prime}(x, s)\right] u_{0}(s) d s \\
& \quad+\int_{x}^{1}\left[L_{1}^{\prime}\left(x, x_{0}\right) R(x, s) / L\left(x, x_{0}\right)-R_{1}^{\prime}(x, s)\right] u_{0}(s) d s
\end{aligned}
$$

for $x>x_{0}$. From (1.28) and (1.29), we conclude that if the equations

$$
J(x) u(x)+G(x)=\int_{0}^{1}\left[R_{1}^{\prime}\left(x, x_{0}\right) K(x, s) / R\left(x, x_{0}\right)-K_{1}^{\prime}(x, s)\right] u(s) d s(1.30)
$$

for $x<x_{0}$, and

$$
J(x) u(x)+G(x)=\int_{0}^{1}\left[L_{1}^{\prime}\left(x, x_{0}\right) K(x, s) / L\left(x, x_{0}\right)-K_{1}^{\prime}(x, s)\right] u(s) d s
$$

for $x>x_{0}$, have unique solutions respectively, then we can obtain the leading order outer solution $u_{0}$ and furthermore determine $c$ in (1.26). In this way we obtain the leading order of the asymptotic expansion of the composite solution of (1.1).

\section{Residual analysis}

To obtain a more accurate solution of (1.1), we first obtain $B_{1}(Z)$ from (1.14) and (1.15):

$$
\begin{aligned}
B_{1}(Z)-\int_{-\infty}^{Z}( & \left.Z \frac{\partial}{\partial x}+S \frac{\partial}{\partial s}\right)\left[L\left(x_{0}, x_{0}\right)-R\left(x_{0}, x_{0}\right)\right] B_{1}(S) d S \\
& =\int_{-\infty}^{Z}\left(Z \frac{\partial}{\partial x}+S \frac{\partial}{\partial s}\right)^{2}\left[L\left(x_{0}, x_{0}\right)-R\left(x_{0}, x_{0}\right)\right] B_{0}(S) / 2 d S
\end{aligned}
$$

for $x<x_{0}$, and

$$
\begin{aligned}
B_{1}(Z)-\int_{Z}^{+\infty}\left(Z \frac{\partial}{\partial x}+S \frac{\partial}{\partial x}\right)\left[L\left(x_{0}, x_{0}\right)-R\left(x_{0}, x_{0}\right)\right] B_{1}(S) d S \\
\quad=\int_{Z}^{+\infty}\left(Z \frac{\partial}{\partial x}+S \frac{\partial}{\partial s}\right)^{2}\left[L\left(x_{0}, x_{0}\right)-R\left(x_{0}, x_{0}\right)\right] B_{0}(S) / 2 d S
\end{aligned}
$$

for $x>x_{0}$. We prove that $v(x, \varepsilon)$, defined as follows,

$$
v(x, \varepsilon)=u_{0}(x)+\alpha(\varepsilon)\left[B_{0}(Z)+\varepsilon^{1 / 2} B_{1}(Z)\right],
$$

approximately satisfies (1.1). First we define $\rho(x, \varepsilon)$ as

$$
\rho(x, \varepsilon)=\varepsilon v(x, \varepsilon)+h(x, \varepsilon)-\int_{0}^{1} K(x, s) v(s, \varepsilon) d s, \quad(0 \leq x \leq 1) .
$$


We substitute (2.3) into (2.4) and derive

$$
\begin{aligned}
\rho(x, \varepsilon)=\varepsilon[ & \left.u_{0}(x)+\alpha(\varepsilon)\left(B_{0}(Z)+\varepsilon^{1 / 2} B_{1}(Z)\right)\right] \\
& +h(x, \varepsilon)-\int_{0}^{1} K(x, s)\left[u_{0}(s)+\alpha(\varepsilon)\left(B_{0}(S)+\varepsilon^{1 / 2} B_{1}(S)\right)\right] d s .
\end{aligned}
$$

If $x \leq x_{0}$, from (1.21), (1.22) and (2.1) we have

$$
\begin{aligned}
\rho(x, \varepsilon)=\varepsilon & u_{0}(x)+h\left(x_{0}, \varepsilon\right)-h_{0}(x)+\int_{-\infty}^{+\infty} R\left(x, x_{0}\right) B_{0}(S) d S \\
& +\varepsilon^{1 / 2} \int_{-\infty}^{+\infty} R\left(x, x_{0}\right) B_{1}(S) d S-\int_{0}^{1} K(x, s)\left[\alpha(\varepsilon) B_{0}(S)\right] d S \\
& +\varepsilon^{1 / 2} \int_{-\infty}^{Z}\left(Z \frac{\partial}{\partial x}+S \frac{\partial}{\partial s}\right)\left[L\left(x_{0}, x_{0}\right)-R\left(x_{0}, x_{0}\right)\right] B_{0}(S) d S \\
& +\varepsilon \int_{-\infty}^{Z}\left(Z \frac{\partial}{\partial x}+S \frac{\partial}{\partial s}\right)\left[L\left(x_{0}, x_{0}\right)-R\left(x_{0}, x_{0}\right)\right] B_{1}(S) d S \\
& +\varepsilon \int_{-\infty}^{+\infty}\left(Z \frac{\partial}{\partial x}+S \frac{\partial}{\partial s}\right)^{2}\left[L\left(x_{0}, x_{0}\right)-R\left(x_{0}, x_{0}\right)\right] B_{0}(S) / 2 d S \\
& -\int_{0}^{1} K(x, s)\left[\alpha(\varepsilon) \varepsilon^{1 / 2} B_{1}(S)\right] d S .
\end{aligned}
$$

If $B_{0}(Z)$ and $B_{1}(Z)$ have the kind of asymptotic forms of (1.26) as $Z \rightarrow \infty$, then we have

$$
\begin{aligned}
& \alpha(\varepsilon) \int_{0}^{1} K(x, s) B_{0}(S) d S \sim \int_{-\infty}^{+\infty} R\left(x, x_{0}\right) B_{0}(S) d S \\
&+\varepsilon^{1 / 2} \int_{-\infty}^{Z}\left(Z \frac{\partial}{\partial x}+S \frac{\partial}{\partial s}\right)\left[L\left(x_{0}, x_{0}\right)-R\left(x_{0}, x_{0}\right)\right] B_{0}(S) d S+0(\varepsilon),
\end{aligned}
$$

and

$$
\int_{0}^{1} K(x, s) B_{1}(S) d S \sim \varepsilon^{1 / 2} \int_{-\infty}^{+\infty} R\left(x, x_{0}\right) B_{1}(S) d S+0(\varepsilon)
$$

Finally we have the estimation of $\rho(x, \varepsilon)$

$$
\rho(x, \varepsilon)=0(\varepsilon)
$$

for $x \leq x_{0}$ as $\varepsilon \rightarrow 0^{+}$. In a similar way, we can derive from (1.23), (1.24) and (2.2) that (2.9) is valid for $x \geq x_{0}$ as $\varepsilon \rightarrow 0^{+}$. By differentiating (2.5), we can obtain the 
representation for $\rho^{\prime}(x, \varepsilon)$ :

$$
\begin{aligned}
\rho^{\prime}(x, \varepsilon)=\varepsilon[ & \left.u_{0}^{\prime}(x)+B_{0}^{\prime}(Z)+\varepsilon^{1 / 2} B_{1}^{\prime}(Z)\right]+h^{\prime}(x, \varepsilon) \\
& -[L(x, x)-R(x, x)]\left\{u_{0}(x)+\alpha(\varepsilon)\left[B_{0}(Z)+\varepsilon^{1 / 2} B_{1}(Z)\right]\right\} \\
& -\int_{0}^{x} L_{1}^{\prime}(x, s)\left\{u_{0}(s)+\alpha(\varepsilon)\left[B_{0}(S)+\varepsilon^{1 / 2} B_{1}(S)\right]\right\} d S \\
& -\int_{0}^{x} R_{1}^{\prime}(x, s)\left\{u_{0}(s)+\alpha(\varepsilon)\left[B_{0}(S)+\varepsilon^{1 / 2} B_{1}(S)\right]\right\} d S .
\end{aligned}
$$

From differentiations of (1.21) and (1.22), using the fast decay of $B_{0}(Z)$ and $B_{1}(Z)$ as $Z \rightarrow \infty$, we can also derive that

$$
\rho^{\prime}(x, \varepsilon)=0\left(\varepsilon^{1 / 2}\right)
$$

and

$$
\int_{0}^{1}\left|\rho^{\prime}(x, \varepsilon)\right| d x=0(\varepsilon)
$$

as $\varepsilon \rightarrow 0^{+}$

\section{Examples}

In this section, we consider some examples. The first example is

$$
\varepsilon u(x)+\exp \left[(x-1 / 2)^{2}\right]+\int_{0}^{x} 2 s u(s) d s+\int_{x}^{1} u(s) d s=0 .
$$

By comparing (3.1) with (1.1), we have

$$
h(x)=\exp \left[(x-1 / 2)^{2}\right]
$$

and

$$
K(x, s)= \begin{cases}-2 s, & s<x \\ -1, & s>x\end{cases}
$$

Since the jump is zero at $x_{0}$, clearly the interior layer is located at $x_{0}=1 / 2$.

The leading order equation for the outer solution is

$$
\exp \left[(x-1 / 2)^{2}\right]+\int_{0}^{x} 2 s u_{0}(s) d s+\int_{x}^{1} u_{0}(s) d s=c_{0},
$$

where

$$
c_{0}=\int_{-\infty}^{+\infty}-B_{0}(S) d S
$$

By differentiating (3.4) we obtain the leading order solution,

$$
u_{0}(x)=-\exp \left[(x-1 / 2)^{2}\right]
$$

By substituting (3.6) into (3.4), $c_{0}$ is obtained:

$$
c_{0}=e^{1 / 4}-2 \int_{0}^{1 / 2} e^{s^{2}} d s .
$$


The leading order equation for the "boundary layer correction" is

$$
B_{0}(Z)=\int_{-\infty}^{Z}-2 S B_{0}(S) d S
$$

for $x<x_{0}$ and

$$
B_{0}(Z)=\int_{Z}^{+\infty} 2 S B_{0}(S) d S
$$

for $x>x_{0}$. We solve (3.8) and (3.9), obtaining

$$
B_{0}(Z)=c_{1} e^{-Z^{2}}
$$

From (3.5) and (3.7) we obtain

$$
c_{1}=\frac{1}{\sqrt{2 \pi}}\left(e^{1 / 4}-2 \int_{0}^{1 / 2} e^{s^{2}} d s\right) .
$$

In this way, we have obtained the asymptotic composite expansion of the solution of $(3.1)$ :

$$
u(x) \sim-\exp \left[(x-1 / 2)^{2}\right]+\varepsilon^{-1 / 2} \frac{1}{\sqrt{2 \pi}}\left(e^{1 / 4}-2 \int_{0}^{1 / 2} e^{s^{2}} d s\right) e^{-Z^{2}} .
$$

The second example is

$$
\varepsilon u(x)+e^{x}+\int_{0}^{x} 2 s u(s) d s+\int_{x}^{1} u(s) d s=0 .
$$

The kernel of the integral equation and the position of the interior layer located are the same as in the first example. But for this case, we can prove that the "boundary layer correction" does not decay fast enough in the outer regions $\left(x<x_{0}\right.$ or $\left.x>x_{0}\right)$. Then the boundary layer correction method can not be applied directly. To overcome this difficulty we use instead the method of matched asymptotic expansions (the general method applied to Fredholm integral equations will be described in another paper). We can show that the leading order of the outer solution is determined in a similar way as that in Example 1. That is, it should satisfy the following equation:

$$
e^{x}+\int_{0}^{x} 2 s u_{0}(s) d s+\int_{x}^{1} u_{0}(s) d s=C_{0},
$$

where $C_{0}$ is represented by

$$
C_{0}=\int_{-\infty}^{+\infty}-B(S) d S
$$

By differentiating (3.14) we obtain the leading order solution

$$
u_{0}(x)=\frac{e^{x}}{1-2 x}
$$

The leading order of the inner equation is

$$
1=\int_{-\infty}^{+\infty}-B(S) d S+\operatorname{Pr} \int_{0}^{1} \frac{e^{S}}{1-2 S} d S
$$


where Pr represents the Cauchy principal-part integral. The next order of inner equation is

$$
B(Z)+e^{1 / 2}=\int_{-\infty}^{Z}-2 S B(S) d S
$$

for $x<x_{0}$ and

$$
B(Z)+e^{1 / 2}=\int_{Z}^{+\infty} 2 S B(S) d S
$$

for $x>x_{0}$. We solve (3.17) and (3.18), obtaining the leading order of the inner solution

$$
B(Z)=\left[C_{1}-e^{1 / 2} \int_{0}^{Z} e^{s^{2}} d s\right] e^{-Z^{2}} .
$$

From (3.19) and (3.20) we obtain

$$
C_{1}=-(1 / \sqrt{\pi})\left[1+\operatorname{Pr} \int_{0}^{1} e^{s} /(1-2 s) d s\right] .
$$

In this way, we can obtain the leading order inner solution of (3.13):

$$
B(Z)=\left\{\frac{-1}{\sqrt{\pi}}\left[1+\operatorname{Pr} \int_{0}^{1} \frac{e^{s}}{1-2 s} d s\right]-e^{1 / 2} \int_{0}^{Z} e^{s^{2}} d s\right\} e^{-Z^{2}} .
$$

We can see that $B(Z)$ does not decay very quickly as $Z \rightarrow \infty$ as we mentioned before. The uniform asymptotic composite expression of the solution of (3.13) can be obtained by adding the outer solution (3.16) and (3.22) (multiplied by $\varepsilon^{-1 / 2}$ ), then subtracting the "common part":

$$
V_{c}(x)=\frac{e^{1 / 2}}{1-2 x}
$$

The result is

$$
u(x) \sim \frac{e^{x}-e^{1 / 2}}{1-2 x}+\varepsilon^{-1 / 2}\left\{\frac{-1}{\sqrt{\pi}}\left[1+\operatorname{Pr} \int_{0}^{1} \frac{e^{s}}{1-2 s} d s\right]-e^{1 / 2} \int_{0}^{Z} e^{s^{2}} d s\right\} e^{-Z^{2}} .
$$

The asymptotic behavior may also be obtained from the exact solution of (3.1) by using the method of steepest decent (the saddle-point method). The details are omitted here.

The method introduced in this paper can be employed in certain studies of singularly perturbed integral equations, as illustrated in Example 1. But the results of the paper are rather special and limited. The restrictions of (1.11), (1.16) and (1.25) are necessary. The most important condition is that the solution should possess an asymptotic expansion of the form of (1.5) with a suitably rapidly decaying layer correction. It can be checked a' posteriori in individual cases. Generally, if the "outer solution" is singular as $x \rightarrow x_{0}$, then our perturbation ansatz may not be applicable. In that case, the method of matched asymptotic expansions may be more useful (see, for example, $[3, \mathrm{p} .6])$. The sufficient conditions to insure the existence of solutions possessing the asymptotic behavior of (1.5) have not been found; work remains to be done in this field. 
Acknowledgement. The author expresses his thanks to Professor R. Haberman of Southern Methodist University for helpful discussions.

\section{References}

1. R. E. O'Malley, Introduction to Singular Perturbations, Academic Press, 1974.

2. J. Kevorkian and J. D. Cole, Perturbation Methods in Applied Mathematics, Springer-Verlag, New York, 1980.

3. C. G. Lange and D. R. Smith, Singular Perturbation Analysis of Integral Equations, Stud. Appl. Math. 79 (1988), 1-63.

4. W. E. Olmstead and J. S. Angell, Singular Perturbated Integral Equations with End Point Boundary layers, SIAM J. Appl. Math. (6) 49 (1989), 1567-1584.

Department of Mathematics and Mechanics, The University of Science and Technology Beijing, Beijing 100083, The People's Republic of China 